\title{
Meat intake and inflammation: prospective analyses in 15,433 participants of the UK Biobank
}

\author{
K. Papier ${ }^{1}$, L. Hartman ${ }^{2}$, T.Y.N. Tong ${ }^{1}$, T.J. Key ${ }^{1}$ and A. Knuppel ${ }^{1}$ \\ ${ }^{1}$ Cancer Epidemiology Unit, Nuffield Department of Population Health, University of Oxford, Richard Doll Building, \\ Oxford, UK and \\ ${ }^{2}$ Medical School, University of Oxford, Oxford, UK
}

Systemic low-grade inflammation has been associated with a higher risk of chronic diseases ${ }^{(1)}$. Meat consumption might play a role in inflammatory processes, but prospective research on the associations of meat intake and markers of systemic inflammation is limited and suggests that the association might be entirely explained by higher adiposity ${ }^{(2)}$. The aim of this study was to assess the prospective association of meat consumption (total, unprocessed red, and processed meat, and poultry) with C-reactive protein (CRP) and total white blood cell count (WBCC) in British adults. A secondary aim was to investigate the role of central adiposity in the association.

The study was based on participants from the UK Biobank cohort study that was established between 2006 and 2010 . Meat intake was determined at baseline using questionnaires ascertaining weekly consumption, categorised into groups of intake based on distribution and daily intakes based on estimated portion sizes. CRP concentrations and WBCC were measured in non-fasting blood samples collected from 15,433 participants in 2012-2013 (median 4.4 years after recruitment). We used multivariable linear regression models to determine the associations of meat consumption categorically, and per 50g/day higher intake with CRP and WBCC. We then reran the analyses adjusted for waist circumference to examine the role of central adiposity.

CRP concentrations were positively associated with total meat intake, ranging from adjusted geometric means of $1.04 \mathrm{mg} / \mathrm{L}(95 \%$ confidence intervals (CI) $1.00-1.09)$ in those who ate meat $<3$ times/week to $1.40 \mathrm{mg} / \mathrm{L}(95 \%$-CI $1.36-1.44)$ in those who ate it $7+$ times/ week (standardised $\beta$ per $50 \mathrm{~g} /$ day higher intake $0.11,95 \%$-CI $0.09-0.13$ for log CRP). Across the same categories, geometric means for WBCC were $6.39 \times 109 / \mathrm{L}(95 \%$-CI $6.30-6.47)$ and $6.68 \times 109 / \mathrm{L}(95 \%$-CI 6.62-6.74; standardised $\beta$ per $50 \mathrm{~g} /$ day higher intake 0.05 , 95\%-CI 0.03, 0.07 for log WBCC). We also observed positive associations with CRP and WBCC when investigating unprocessed red, processed meat and poultry intake, separately. When adjusting for central adiposity all associations were attenuated (e.g. for $50 \mathrm{~g} /$ day higher intake of total meat, the standardised $\beta 0.08,95 \%$-CI $0.06,0.10$ for log CRP, and $0.03,95 \%-\mathrm{CI} 0.01,0.05$ for $\log$ WBCC).

This is the largest prospective study to investigate associations of meat intake with inflammatory markers. The findings suggest that total meat intake, including unprocessed red meat, processed meat and poultry, is associated with higher CRP and WBCC, partly explained by increased central adiposity in those with higher meat intakes.

\section{Acknowledgements}

This research has been conducted using the UK Biobank (UKB) resource under application number 24494. We thank all participants, researchers and support staff who make the study possible, and Aurora Perez-Cornago (Cancer Epidemiology Unit, Nuffield Department of Population Health, University of Oxford) for acquiring the data.

\section{References}

1. The Emerging Risk Factors Collaboration (2010) Lancet 375, 132-140.

2. Chai W, Morimoto Y, Cooney RV et al. (2017) J Am Coll Nutr 36, 378-385. 